Journal of Mathematics and Statistics 3 (4): 211-219, 2007

ISSN 1549-3644

C 2007 Science Publications

\title{
Reliability Parameters of a Power Generating System with Shared Load
}

\author{
${ }^{1}$ Ritu Gupta, ${ }^{2}$ S.K. Mittal and ${ }^{1}$ C.M. Batra \\ ${ }^{1}$ Krishna Institute of Engineering and Technology, Ghaziabad, UP, India \\ ${ }^{2}$ M.M. (PG) College, Modinagar, India
}

\begin{abstract}
This study presents a model, based on power generating system with shared load. The whole generating system consists of three subsystems viz: subsystem A, subsystem B and subsystem C. The subsystem A consists of one generating unit and one inbuilt transformer. The subsystem B also contains the same units and is connected in parallel to subsystem A. The output of this power system goes through the subsystem $\mathrm{C}$ that consists of one outer transformer and which may be further distributed as desired. The system has three types of states, viz: normal, degraded and failed. All types of failure rates and repair rates of inbuilt transformers are exponential while other repair rates are distributed quite generally. Supplementary Variable Technique has been employed to obtain various state probabilities and then the reliability parameters have been evaluated for the whole generating system.
\end{abstract}

Key words: Reliability, availability, MTTF, SVT

\section{INRODUCTION}

Electric energy demand has been rapidly increasing all over the world. This is attributed to greater industrialization and large-scale use of electric energy for agricultural purpose. The demand is likely to increase exponentially for many more decades to come. There are no signs of saturation in the foreseeable future. Electric supply authorities are likely to pay more attention to improve the utilization of generating equipment. The reliability of electric supply in India is very low. The public is likely to become more and more conscious of its rights to get uninterrupted supply at proper voltage. This would force the electric supply undertakings to analyze the system and take corrective measures to improve reliability.

Keeping these points in view, the author has considered a mathematical model by which the reliability of the generating system can be improved. The whole generating system consists of three subsystems viz, subsystem A, subsystem B and subsystem C. The subsystem A consists of one generating unit and one inbuilt transformer. The subsystem B, arranged in parallel with subsystem A, is a redundant system and also consists of one generating unit and one inbuilt transformer. The output of these two subsystems goes through the subsystem $\mathrm{C}$ that consists of one outer transformer and the electric supply may be further distributed from this subsystem $\mathrm{C}$ as desired. The power required at subsystem
C is shared by two subsystems, A and B which will increase the availability of power in comparison to that which is, instead, produced by a single subsystem A. Supplementary Variable technique has been employed to evaluate various reliability parameters of the generating system. The mathematical model of the whole system is shown in the state transition diagram.

\section{ASSUMPTIONS}

- At time $\mathrm{t}=0$, the system is in operable state.

- The subsystem B works as the redundant unit.

- $\quad$ All the failure rates and repair rates of unit A2 and unit B2 (inbuilt transformers) are exponential while other repair rates are distributed quite generally.

- The failure rate of all units is distinct.

- After the complete breakdown of the system, the repair rate is assumed as same.

- The system is in degraded state after the failure of either unit of subsystem A or subsystem B, or completely subsystem A or subsystem B.

- All the units recover their functioning perfectly after repair.

Formulation of the model: The probabilities given above are mutually exclusive and provide the complete markovian characteristic of the process. Therefore, using continuity arguments and elementary probability considerations, one get the following difference

Corresponding Author: Ritu Gupta, Department of Mathematics, Krishna Institute of Engineering \& Technology, 13 KM Stone, Ghaziabad-Meerut Road, Ghaziabad-201206, UP, India 
differential equations governing the stochastic behaviour of the complex system, which is discrete in space and continuous in time:

$$
\begin{aligned}
& \left(\frac{d}{d t}+\lambda_{a}+\lambda_{b}+\lambda_{1}+\lambda_{2}+\lambda\right) P_{1}(t)= \\
& \int \mu_{1}(x) P_{2}(x, t) d x+\int \mu(z) P_{4}(z, t) d z \\
& +\int \mu_{1}(\mathrm{x}) \mathrm{P}_{3}(\mathrm{x}, \mathrm{t}) \mathrm{dx}+\int \mu(\mathrm{z}) \mathrm{P}_{8}(\mathrm{z}, \mathrm{t}) \mathrm{dz}+ \\
& \int \mu(\mathrm{z}) \mathrm{P}_{7}(\mathrm{z}, \mathrm{t}) \mathrm{dz}+\int \mu_{2}(\mathrm{y}) \mathrm{P}_{6}(\mathrm{y}, \mathrm{t}) \mathrm{dy} \\
& +\int \mu(\mathrm{z}) \mathrm{P}_{9}(\mathrm{z}, \mathrm{t}) \mathrm{dz}+v \mathrm{P}_{5}(\mathrm{t})+v \mathrm{P}_{11}(\mathrm{t})+ \\
& \int \mu_{2}(y) P_{12}(y, t) d y+\int \mu(z) P_{10}(z, t) d z \\
& +\int \mu(z) P_{13}(z, t) d z+\int \mu(z) P_{14}(z, t) d z+ \\
& \int \mu(\mathrm{z}) \mathrm{P}_{15}(\mathrm{z}, \mathrm{t}) \mathrm{dz}+\int \mu(\mathrm{z}) \mathrm{P}_{16}(\mathrm{z}, \mathrm{t}) \mathrm{dz} \\
& +\int \mu(\mathrm{z}) \mathrm{P}_{17}(\mathrm{z}, \mathrm{t}) \mathrm{dz} \\
& \left(\frac{\partial}{\partial x}+\frac{\partial}{\partial t}+\lambda+\lambda_{b}+\mu_{1}(x)\right) P_{2}(x, t)=0 \\
& \left(\frac{\partial}{\partial \mathrm{x}}+\frac{\partial}{\partial \mathrm{t}}+\lambda_{\mathrm{a}}+\lambda+\mu_{1}(\mathrm{x})\right) \mathrm{P}_{3}(\mathrm{x}, \mathrm{t})=0 \\
& \left(\frac{\partial}{\partial z}+\frac{\partial}{\partial t}+\mu(\mathrm{z})\right) \mathrm{P}_{4}(\mathrm{z}, \mathrm{t})=0 \\
& \left(\frac{\partial}{\partial \mathrm{t}}+v+\lambda_{2}+\lambda_{\mathrm{b}}+\lambda+\lambda_{\mathrm{a}}\right) \mathrm{P}_{5}(\mathrm{t})=\lambda_{1} \mathrm{P}_{1}(\mathrm{t}) \\
& \left(\frac{\partial}{\partial y}+\frac{\partial}{\partial t}+\lambda_{b}+\lambda_{2}+\mu_{2}(y)+\lambda\right) P_{6}(y, t)=0 \\
& \left(\frac{\partial}{\partial \mathrm{z}}+\frac{\partial}{\partial \mathrm{t}}+\mu(\mathrm{z})\right) \mathrm{P}_{7}(\mathrm{z}, \mathrm{t})=0 \\
& \left(\frac{\partial}{\partial z}+\frac{\partial}{\partial t}+\mu(\mathrm{z})\right) \mathrm{P}_{8}(\mathrm{z}, \mathrm{t})=0 \\
& \left(\frac{\partial}{\partial z}+\frac{\partial}{\partial t}+\mu(z)\right) P_{9}(z, t)=0 \\
& \left(\frac{\partial}{\partial \mathrm{z}}+\frac{\partial}{\partial \mathrm{t}}+\mu(\mathrm{z})\right) \mathrm{P}_{10}(\mathrm{z}, \mathrm{t})=0 \\
& \left(\frac{\mathrm{d}}{\mathrm{dt}}+\lambda+\lambda_{\mathrm{b}}+\lambda_{\mathrm{a}}+\lambda_{1}+v\right) \mathrm{P}_{11}(\mathrm{x}, \mathrm{t})=\lambda_{2} \mathrm{P}_{1}(\mathrm{t}) \\
& \left(\frac{\partial}{\partial \mathrm{y}}+\frac{\partial}{\partial \mathrm{t}}+\lambda_{\mathrm{a}}+\lambda_{1}+\mu_{2}(\mathrm{y})+\lambda\right) \mathrm{P}_{12}(\mathrm{y}, \mathrm{t})=0 \\
& \left(\frac{\partial}{\partial \mathrm{z}}+\frac{\partial}{\partial \mathrm{t}}+\mu(\mathrm{z})\right) \mathrm{P}_{13}(\mathrm{z}, \mathrm{t})=0
\end{aligned}
$$

$$
\begin{aligned}
& \left(\frac{\partial}{\partial z}+\frac{\partial}{\partial t}+\mu(z)\right) P_{14}(z, t)=0 \\
& \left(\frac{\partial}{\partial z}+\frac{\partial}{\partial t}+\mu(z)\right) P_{15}(z, t)=0 \\
& \left(\frac{\partial}{\partial z}+\frac{\partial}{\partial t}+\mu(z)\right) P_{16}(z, t)=0 \\
& \left(\frac{\partial}{\partial z}+\frac{\partial}{\partial t}+\mu(z)\right) P_{17}(z, t)=0
\end{aligned}
$$

Boundary conditions:

$$
\begin{gathered}
\mathrm{P}_{2}(0, \mathrm{t})=\lambda_{\mathrm{a}} \mathrm{P}_{1}(\mathrm{t}) \\
\mathrm{P}_{3}(0, \mathrm{t})=\lambda_{\mathrm{b}} \mathrm{P}_{1}(\mathrm{t}) \\
\mathrm{P}_{4}(0, \mathrm{t})=\lambda_{\mathrm{a}} \int \mathrm{P}_{3}(\mathrm{x}, \mathrm{t}) \mathrm{dx}+\lambda_{\mathrm{b}} \int \mathrm{P}_{2}(\mathrm{x}, \mathrm{t}) \mathrm{d} \mathrm{x} \\
\mathrm{P}_{6}(0, \mathrm{t})=\lambda_{\mathrm{a}} \mathrm{P}_{5}(\mathrm{t}) \\
\mathrm{P}_{7}(0, \mathrm{t})=\lambda_{\mathrm{b}} \int \mathrm{P}_{6}(\mathrm{y}, \mathrm{t}) \mathrm{dy} \\
\mathrm{P}_{8}(0, \mathrm{t})=\lambda_{2} \int \mathrm{P}_{6}(\mathrm{y}, \mathrm{t}) \mathrm{dy} \\
\mathrm{P}_{9}(0, \mathrm{t})=\lambda_{2} \mathrm{P}_{5}(\mathrm{t}) \\
\mathrm{P}_{10}(0, \mathrm{t})=\lambda_{\mathrm{b}} \mathrm{P}_{5}(\mathrm{t}) \\
\mathrm{P}_{12}(0, \mathrm{t})=\lambda_{\mathrm{b}} \mathrm{P}_{11}(\mathrm{t}) \\
\mathrm{P}_{13}(0, \mathrm{t})=\lambda_{1} \int \mathrm{P}_{12}(\mathrm{y}, \mathrm{t}) \mathrm{dy} \\
\mathrm{P}_{14}(0, \mathrm{t})=\lambda_{\mathrm{a}} \int \mathrm{P}_{12}(\mathrm{y}, \mathrm{t}) \mathrm{dy} \\
\mathrm{P}_{15}(0, \mathrm{t})=\lambda_{1} \mathrm{P}_{11}(\mathrm{t}) \\
\mathrm{P}_{16}(0, \mathrm{t})=\lambda_{\mathrm{a}} \mathrm{P}_{11}(\mathrm{t}) \\
\mathrm{P}_{17}(0, \mathrm{t})=\lambda \int \mathrm{P}_{2}(\mathrm{x}, \mathrm{t}) \mathrm{dx}+\lambda \int \mathrm{P}_{3}(\mathrm{x}, \mathrm{t}) \mathrm{dx}+ \\
\lambda \int \mathrm{P}_{6}(\mathrm{x}, \mathrm{t}) \mathrm{dx}+\lambda \int \mathrm{P}_{12}(\mathrm{x}, \mathrm{t}) \mathrm{dx}+\lambda \mathrm{P}_{1}(\mathrm{t})+ \\
\lambda \mathrm{P}_{11}(\mathrm{t})+\lambda \mathrm{P}_{5}(\mathrm{t})
\end{gathered}
$$

Initial conditions: $P_{1}(0)$ and other state probabilities are zero.

Solution of the model: Taking Laplace Transform of Eq. 1-31 and on further simplification one may obtain:

$$
\begin{gathered}
\overline{\mathrm{P}_{1}}(\mathrm{~s})=\frac{1}{\mathrm{~A}(\mathrm{~s})} \\
\overline{\mathrm{P}_{2}}(\mathrm{~s})=\lambda_{\mathrm{a}} \mathrm{k}_{1}(\mathrm{~s}) \mathrm{P}_{1}(\mathrm{~s}) \\
\overline{\mathrm{P}_{3}}(\mathrm{~s})=\lambda_{\mathrm{b}} \mathrm{k}_{2}(\mathrm{~s}) \mathrm{P}_{1}(\mathrm{~s})
\end{gathered}
$$




$$
\begin{aligned}
& \overline{\mathrm{P}_{4}}(\mathrm{~s})=\lambda_{\mathrm{a}} \lambda_{\mathrm{b}} \mathrm{k}_{4}(\mathrm{~s}) \mathrm{k}_{3}(\mathrm{~s}) \mathrm{P}_{1}(\mathrm{~s}) \\
& \overline{\mathrm{P}_{5}}(\mathrm{~s})=\frac{\lambda_{1}}{\left(\mathrm{~s}+\lambda+\lambda_{\mathrm{a}}+\lambda_{\mathrm{b}}+\lambda_{2}+v\right)} \mathrm{P}_{1}(\mathrm{~s}) \\
& \overline{\mathrm{P}_{6}}(\mathrm{~s})=\mathrm{k}_{6}(\mathrm{~s}) \mathrm{P}_{1}(\mathrm{~s}) \\
& \overline{\mathrm{P}_{7}}(\mathrm{~s})=\mathrm{k}_{7}(\mathrm{~s}) \mathrm{k}_{3}(\mathrm{~s}) \mathrm{P}_{1}(\mathrm{~s}) \\
& \overline{\mathrm{P}_{8}}(\mathrm{~s})=\mathrm{k}_{8}(\mathrm{~s}) \mathrm{k}_{3}(\mathrm{~s}) \mathrm{P}_{1}(\mathrm{~s}) \\
& \overline{\mathrm{P}_{9}}(\mathrm{~s})=\mathrm{k}_{9}(\mathrm{~s}) \mathrm{k}_{3}(\mathrm{~s}) \mathrm{P}_{1}(\mathrm{~s}) \\
& \overline{\mathrm{P}_{10}}(\mathrm{~s})=\mathrm{k}_{11}(\mathrm{~s}) \mathrm{P}_{1}(\mathrm{~s}) \\
& \overline{\mathrm{P}_{11}}(\mathrm{~s})=\frac{\lambda_{2}}{\left(\mathrm{~s}+\lambda+\lambda_{\mathrm{a}}+\lambda_{\mathrm{b}}+\lambda_{1}+v\right)} \mathrm{P}_{1}(\mathrm{~s}) \\
& \overline{\mathrm{P}_{12}}(\mathrm{~s})=\mathrm{k}_{12}(\mathrm{~s}) \mathrm{P}_{1}(\mathrm{~s}) \\
& \overline{\mathrm{P}_{13}}(\mathrm{~s})=\mathrm{k}_{13}(\mathrm{~s}) \mathrm{k}_{3}(\mathrm{~s}) \mathrm{P}_{1}(\mathrm{~s}) \\
& \overline{\mathrm{P}_{14}}(\mathrm{~s})=\mathrm{k}_{14}(\mathrm{~s}) \mathrm{k}_{3}(\mathrm{~s}) \mathrm{P}_{1}(\mathrm{~s}) \\
& \overline{\mathrm{P}_{15}}(\mathrm{~s})=\mathrm{k}_{15}(\mathrm{~s}) \mathrm{k}_{3}(\mathrm{~s}) \mathrm{P}_{1}(\mathrm{~s}) \\
& \overline{\mathrm{P}_{16}}(\mathrm{~s})=\mathrm{k}_{16}(\mathrm{~s}) \mathrm{k}_{3}(\mathrm{~s}) \mathrm{P}_{1}(\mathrm{~s}) \\
& \overline{\mathrm{P}_{17}}(\mathrm{~s})=\mathrm{k}_{17}(\mathrm{~s}) \mathrm{k}_{3}(\mathrm{~s}) \mathrm{P}_{1}(\mathrm{~s})
\end{aligned}
$$

where,

$$
\begin{gathered}
\mathrm{A}(\mathrm{s})=\left(\mathrm{s}+\lambda_{\mathrm{a}}+\lambda_{\mathrm{b}}+\lambda_{1}+\lambda_{2}+\lambda\right)-\mathrm{C}_{1}(\mathrm{~s})- \\
\mathrm{C}_{2}(\mathrm{~s})-\mathrm{C}_{3}(\mathrm{~s})-\mathrm{C}_{4}(\mathrm{~s})-\mathrm{C}_{5}(\mathrm{~s})-\mathrm{C}_{6}(\mathrm{~s})-\mathrm{C}_{7}(\mathrm{~s})- \\
\mathrm{C}_{8}(\mathrm{~s})-\mathrm{C}_{9}(\mathrm{~s})-\mathrm{C}_{10}(\mathrm{~s})-\mathrm{C}_{11}(\mathrm{~s})-\mathrm{C}_{12}(\mathrm{~s})-\mathrm{C}_{13}(\mathrm{~s})-\mathrm{C}_{14}(\mathrm{~s}) \\
\mathrm{k}_{1}(\mathrm{~s})=\frac{1-\overline{\mathrm{S}}_{1}\left(\mathrm{~s}+\lambda+\lambda_{\mathrm{b}}\right)}{\mathrm{s}+\lambda+\lambda_{\mathrm{b}}} \\
\mathrm{k}_{2}(\mathrm{~s})=\frac{1-\overline{\mathrm{S}}_{1}\left(\mathrm{~s}+\lambda+\lambda_{\mathrm{a}}\right)}{\mathrm{s}+\lambda+\lambda_{\mathrm{a}}}, \mathrm{k}_{3}(\mathrm{~s})=\frac{1-\overline{\mathrm{S}}_{\mu}(\mathrm{s})}{\mathrm{s}} \\
\mathrm{k}_{4}(\mathrm{~s})=\left(\mathrm{k}_{2}(\mathrm{~s})+\mathrm{k}_{1}(\mathrm{~s})\right)
\end{gathered}
$$

$$
\begin{gathered}
\mathrm{k}_{5}(\mathrm{~s})=\frac{1-\overline{\mathrm{S}}_{2}\left(\mathrm{~s}+\lambda+\lambda_{2}+\lambda_{\mathrm{b}}\right)}{\mathrm{s}+\lambda+\lambda_{\mathrm{b}}+\lambda_{2}}, \\
\mathrm{k}_{6}(\mathrm{~s})=\frac{\lambda_{\mathrm{a}} \lambda_{1}}{\left(\mathrm{~s}+v+\lambda_{\mathrm{a}}+\lambda_{\mathrm{b}}+\lambda_{2}+\lambda\right)} \mathrm{k}_{5}(\mathrm{~s}) \\
\mathrm{k}_{7}(\mathrm{~s})=\lambda_{\mathrm{b}} \mathrm{k}_{6}(\mathrm{~s}), \mathrm{k}_{8}(\mathrm{~s})=\lambda_{2} \mathrm{k}_{6}(\mathrm{~s}) \\
\mathrm{k}_{9}(\mathrm{~s})=\frac{\lambda_{2} \lambda_{1}}{\left(\mathrm{~s}+v+\lambda_{\mathrm{a}}+\lambda_{\mathrm{b}}+\lambda_{2}+\lambda\right)} \\
\mathrm{k}_{10}(\mathrm{~s})=\frac{\lambda_{\mathrm{b}} \lambda_{1}}{\left(\mathrm{~s}+v+\lambda_{\mathrm{a}}+\lambda_{\mathrm{b}}+\lambda_{2}+\lambda\right)} \\
\mathrm{k}_{11}(\mathrm{~s})=\frac{1-\overline{\mathrm{S}}_{2}\left(\mathrm{~s}+\lambda+\lambda_{1}+\lambda_{\mathrm{a}}\right)}{\mathrm{s}+\lambda+\lambda_{\mathrm{a}}+\lambda_{1}} \\
\mathrm{k}_{12}(\mathrm{~s})=\frac{\lambda_{\mathrm{b}} \lambda_{2}}{\left(\mathrm{~s}+\mathrm{v}+\lambda_{\mathrm{a}}+\lambda_{\mathrm{b}}+\lambda_{1}+\lambda\right)} \mathrm{k}_{11}(\mathrm{~s}) \\
\mathrm{k}_{13}(\mathrm{~s})=\lambda_{1} \mathrm{k}_{12}(\mathrm{~s}), \mathrm{k}_{14}(\mathrm{~s})=\lambda_{\mathrm{a}} \mathrm{k}_{12}(\mathrm{~s}) \\
\mathrm{k}_{15}(\mathrm{~s})=\frac{\lambda_{\mathrm{a}} \lambda_{2}}{\left(\mathrm{~s}+v+\lambda_{\mathrm{a}}+\lambda_{\mathrm{b}}+\lambda_{1}+\lambda\right)}
\end{gathered}
$$$$
\mathrm{k}_{17}(\mathrm{~s})=\lambda \mathrm{k}_{1}(\mathrm{~s}) \lambda_{\mathrm{a}}+\lambda \lambda_{\mathrm{b}} \mathrm{k}_{2}(\mathrm{~s})+\lambda+
$$$$
\frac{\lambda \lambda_{2}}{\left(\mathrm{~s}+v+\lambda_{\mathrm{a}}+\lambda_{\mathrm{b}}+\lambda_{1}+\lambda\right)}
$$$$
+\frac{\lambda \lambda_{1}}{\left(\mathrm{~s}+v+\lambda_{\mathrm{a}}+\lambda_{\mathrm{b}}+\lambda_{2}+\lambda\right)}+\lambda \mathrm{k}_{12}(\mathrm{~s})+\lambda \mathrm{k}_{6}(\mathrm{~s})
$$$$
\mathrm{C}_{1}(\mathrm{~s})=\lambda_{\mathrm{a}} \overline{\mathrm{S}}_{1}\left(\mathrm{~s}+\lambda+\lambda_{\mathrm{b}}\right),
$$$$
\mathrm{C}_{2}(\mathrm{~s})=\lambda_{\mathrm{a}} \lambda_{\mathrm{b}}\left(\mathrm{k}_{2}(\mathrm{~s})+\mathrm{k}_{1}(\mathrm{~s})\right) \overline{\mathrm{S}}_{\mu}(\mathrm{s})
$$$$
\mathrm{C}_{3}(\mathrm{~s})=\lambda_{\mathrm{b}} \overline{\mathrm{S}}_{1}\left(\mathrm{~s}+\lambda+\lambda_{\mathrm{a}}\right), \mathrm{C}_{4}(\mathrm{~s})=\mathrm{k}_{6}(\mathrm{~s}) \lambda_{2} \overline{\mathrm{S}}_{\mu}(\mathrm{s}),
$$$$
\mathrm{C}_{5}(\mathrm{~s})=\mathrm{k}_{6}(\mathrm{~s}) \lambda_{\mathrm{b}} \overline{\mathrm{S}}_{\mu}(\mathrm{s})
$$$$
\begin{gathered}
\mathrm{C}_{6}(\mathrm{~s})=\frac{\lambda_{\mathrm{a}} \lambda_{1}}{\left(\mathrm{~s}+v+\lambda_{\mathrm{a}}+\lambda_{\mathrm{b}}+\lambda_{2}+\lambda\right)} \overline{\mathrm{S}}_{2}\left(\mathrm{~s}+\lambda+\lambda_{2}+\lambda_{\mathrm{b}}\right) \\
\mathrm{C}_{7}(\mathrm{~s})=\frac{\lambda_{2} \lambda_{1}}{\left(\mathrm{~s}+v+\lambda_{\mathrm{a}}+\lambda_{\mathrm{b}}+\lambda_{2}+\lambda\right)} \overline{\mathrm{S}}_{\mu}(\mathrm{s})
\end{gathered}
$$ 


$$
\begin{gathered}
\mathrm{C}_{8}(\mathrm{~s})=\frac{\lambda_{\mathrm{b}} \lambda_{2}}{\left(\mathrm{~s}+v+\lambda_{\mathrm{a}}+\lambda_{\mathrm{b}}+\lambda_{1}+\lambda\right)} \overline{\mathrm{S}}_{2}\left(\mathrm{~s}+\lambda_{1}+\lambda_{\mathrm{a}}+\lambda\right) \\
\mathrm{C}_{9}(\mathrm{~s})=\frac{\lambda_{\mathrm{b}} \lambda_{1}}{\left(\mathrm{~s}+v+\lambda_{\mathrm{a}}+\lambda_{\mathrm{b}}+\lambda_{2}+\lambda\right)} \overline{\mathrm{S}}_{\mu}(\mathrm{s}) \\
\mathrm{C}_{10}(\mathrm{~s})=\mathrm{k}_{12}(\mathrm{~s}) \lambda_{1} \overline{\mathrm{S}}_{\mu}(\mathrm{s}), \mathrm{C}_{11}(\mathrm{~s})=\mathrm{k}_{12}(\mathrm{~s}) \lambda_{\mathrm{a}} \overline{\mathrm{S}}_{\mu}(\mathrm{s}) \\
\mathrm{C}_{12}(\mathrm{~s})=\frac{\lambda_{1} \lambda_{2}}{\left(\mathrm{~s}+v+\lambda_{\mathrm{a}}+\lambda_{\mathrm{b}}+\lambda_{1}+\lambda\right)} \overline{\mathrm{S}}_{\mu}(\mathrm{s}) \\
\mathrm{C}_{13}(\mathrm{~s})=\frac{\lambda_{\mathrm{a}} \lambda_{2}}{\left(\mathrm{~s}+v+\lambda_{\mathrm{a}}+\lambda_{\mathrm{b}}+\lambda_{1}+\lambda\right)} \overline{\mathrm{S}}_{\mu}(\mathrm{s}) \\
\lambda \overline{\mathrm{S}}_{\mu}(\mathrm{s})\left(\begin{array}{l}
\mathrm{k}_{1}(\mathrm{~s}) \lambda_{\mathrm{a}}+\lambda_{\mathrm{b}} \mathrm{k}_{2}(\mathrm{~s})+1+ \\
\frac{\lambda_{2}}{\left(\mathrm{~s}+v+\lambda_{\mathrm{a}}+\lambda_{\mathrm{b}}+\lambda_{2}+\lambda\right)} \\
+\frac{\lambda_{1}}{\left(\mathrm{~s}+v+\lambda_{\mathrm{a}}+\lambda_{\mathrm{b}}+\lambda_{2}+\lambda\right)} \\
+\mathrm{k}_{12}(\mathrm{~s})+\mathrm{k}_{6}(\mathrm{~s})
\end{array}\right)
\end{gathered}
$$

\section{OPERATIONAL AVAILABILITY AND NON AVAILABILITY}

The Laplace Transform of the probabilities that the system is in operable and down state at time $t$, are given as follows:

$$
\begin{aligned}
& \overline{\mathrm{P}}_{\mathrm{up}}(\mathrm{s})=\overline{\mathrm{P}}_{1}(\mathrm{~s})+\overline{\mathrm{P}}_{2}(\mathrm{~s})+\overline{\mathrm{P}}_{3}(\mathrm{~s})+\overline{\mathrm{P}}_{5}(\mathrm{~s})+\overline{\mathrm{P}}_{6}(\mathrm{~s})+\overline{\mathrm{P}}_{11}(\mathrm{~s})+ \\
& \overline{\mathrm{P}}_{12}(\mathrm{~s})=\mathrm{P}(\mathrm{s})\left(\begin{array}{l}
1+\lambda_{\mathrm{a}} \mathrm{k}_{1}(\mathrm{~s})+\lambda_{\mathrm{b}} \mathrm{k}_{2}(\mathrm{~s})+ \\
\frac{\lambda_{1}}{\left(\mathrm{~s}+v+\lambda_{\mathrm{a}}+\lambda_{\mathrm{b}}+\lambda_{2}+\lambda\right)}+\mathrm{k}_{6}(\mathrm{~s}) \\
+\frac{\lambda_{2}}{\left(\mathrm{~s}+v+\lambda_{\mathrm{a}}+\lambda_{\mathrm{b}}+\lambda_{1}+\lambda\right)}+\mathrm{k}_{12}(\mathrm{~s})
\end{array}\right) \\
& \overline{\mathrm{P}}_{\text {down }}(\mathrm{s})=\overline{\mathrm{P}}_{4}(\mathrm{~s})+\overline{\mathrm{P}}_{7}(\mathrm{~s})+\overline{\mathrm{P}}_{8}(\mathrm{~s})+\overline{\mathrm{P}}_{9}(\mathrm{~s})+ \\
& \overline{\mathrm{P}}_{10}(\mathrm{~s})+\overline{\mathrm{P}}_{13}(\mathrm{~s})+\overline{\mathrm{P}}_{14}(\mathrm{~s})+\overline{\mathrm{P}}_{15}(\mathrm{~s})+\overline{\mathrm{P}}_{16}(\mathrm{~s})+\overline{\mathrm{P}}_{17}(\mathrm{~s})= \\
& \mathrm{P}_{1}(\mathrm{~s}) \mathrm{k}_{3}(\mathrm{~s})\left(\begin{array}{l}
\lambda_{\mathrm{a}} \lambda_{\mathrm{b}} \mathrm{k}_{4}(\mathrm{~s})+\mathrm{k}_{7}(\mathrm{~s})+\mathrm{k}_{8}(\mathrm{~s})+\mathrm{k}_{9}(\mathrm{~s})+\mathrm{k}_{10}(\mathrm{~s})+ \\
\mathrm{k}_{13}(\mathrm{~s})+\mathrm{k}_{14}(\mathrm{~s})+\mathrm{k}_{15}(\mathrm{~s})+\mathrm{k}_{16}(\mathrm{~s})+\mathrm{k}_{17}(\mathrm{~s})
\end{array}\right)
\end{aligned}
$$

It is worth noticing that:

$$
\overline{\mathrm{P}}_{\text {up }}(\mathrm{s})+\overline{\mathrm{P}}_{\text {down }}(\mathrm{s})=\frac{1}{\mathrm{~s}}
$$

Ergodic behaviour: Using Abel's lemma is Laplace transform, viz,

$\lim _{\mathrm{s} \rightarrow 0} \overline{\mathrm{f}}(\mathrm{s})=\lim _{\mathrm{t} \rightarrow \infty} . \mathrm{f}(\mathrm{t})=\mathrm{f}($ say $)$

provided that the limit on the RHS exists, the time independent up and down state probabilities are obtained as follows:

$$
\begin{gathered}
\overline{\mathrm{P}}_{\text {up }}=\frac{1}{\mathrm{~A}^{\prime}(0)}\left\{\begin{array}{l}
1+\lambda_{\mathrm{a}} \mathrm{k}_{1}(0)+\lambda_{\mathrm{b}} \mathrm{k}_{2}(0)+ \\
\frac{\lambda_{1}}{\left(v+\lambda_{\mathrm{a}}+\lambda_{\mathrm{b}}+\lambda_{2}+\lambda\right)}+\mathrm{k}_{6}(0)+ \\
\frac{\lambda_{2}}{\left(v+\lambda_{\mathrm{a}}+\lambda_{\mathrm{b}}+\lambda_{1}+\lambda\right)}+\mathrm{k}_{12}(0)
\end{array}\right\} \\
\overline{\mathrm{P}}_{\text {down }}=\frac{\mathrm{M}_{\mu}}{\mathrm{A}^{\prime}(0)}\left\{\begin{array}{l}
\lambda_{\mathrm{a}} \lambda_{\mathrm{b}} \mathrm{k}_{4}(0)+\mathrm{k}_{7}(0)+\mathrm{k}_{8}(0)+ \\
\mathrm{k}_{9}(0)+\mathrm{k}_{10}(0)+\mathrm{k}_{13}(0)+\mathrm{k}_{14}(0)+ \\
\mathrm{k}_{15}(0)+\mathrm{k}_{16}(0)+\mathrm{k}_{17}(0)
\end{array}\right\}
\end{gathered}
$$

Particular Case: When all repairs follow exponential distribution

$$
\text { Setting } \quad \overline{\mathrm{S}}_{\mu}=\frac{\mu}{\mathrm{s}+\mu}, \quad \overline{\mathrm{S}}_{\mathrm{i}}=\frac{\mu_{\mathrm{i}}}{\mathrm{s}+\mu_{\mathrm{i}}}, \text { where } \mathrm{I}=1,2
$$

$$
\overline{\mathrm{P}_{1}}(\mathrm{~s})=\frac{1}{\mathrm{~g}_{1}(\mathrm{~s})}
$$

$$
\overline{\mathrm{P}_{2}}(\mathrm{~s})=\mathrm{g}_{2}(\mathrm{~s}) \mathrm{P}_{1}(\mathrm{~s})
$$

$$
\overline{\mathrm{P}_{3}}(\mathrm{~s})=\mathrm{g}_{3}(\mathrm{~s}) \mathrm{P}_{1}(\mathrm{~s})
$$

$$
\overline{\mathrm{P}_{4}}(\mathrm{~s})=\mathrm{g}_{4}(\mathrm{~s}) \mathrm{P}_{1}(\mathrm{~s})
$$

$$
\overline{\mathrm{P}_{5}}(\mathrm{~s})=\mathrm{g}_{5}(\mathrm{~s}) \mathrm{P}_{1}(\mathrm{~s})
$$

$$
\overline{\mathrm{P}_{6}}(\mathrm{~s})=\mathrm{g}_{6}(\mathrm{~s}) \mathrm{P}_{1}(\mathrm{~s})
$$

$\overline{\mathrm{P}_{7}}(\mathrm{~s})=\mathrm{g}_{7}(\mathrm{~s}) \mathrm{P}_{1}(\mathrm{~s})$

$$
\overline{\mathrm{P}_{8}}(\mathrm{~s})=\mathrm{g}_{8}(\mathrm{~s}) \mathrm{P}_{1}(\mathrm{~s})
$$




$$
\begin{aligned}
& \overline{\mathrm{P}_{9}}(\mathrm{~s})=\mathrm{g}_{9}(\mathrm{~s}) \mathrm{P}_{1}(\mathrm{~s}) \\
& \overline{\mathrm{P}_{10}}(\mathrm{~s})=\mathrm{g}_{10}(\mathrm{~s}) \mathrm{P}_{1}(\mathrm{~s}) \\
& \overline{\mathrm{P}_{11}}(\mathrm{~s})=\mathrm{g}_{11}(\mathrm{~s}) \mathrm{P}_{1}(\mathrm{~s}) \\
& \overline{\mathrm{P}_{12}}(\mathrm{~s})=\mathrm{g}_{12}(\mathrm{~s}) \mathrm{P}_{1}(\mathrm{~s}) \\
& \overline{\mathrm{P}_{13}}(\mathrm{~s})=\mathrm{g}_{13}(\mathrm{~s}) \mathrm{P}_{1}(\mathrm{~s}) \\
& \overline{\mathrm{P}_{14}}(\mathrm{~s})=\mathrm{g}_{14}(\mathrm{~s}) \mathrm{P}_{1}(\mathrm{~s}) \\
& \overline{\mathrm{P}_{15}}(\mathrm{~s})=\mathrm{g}_{15}(\mathrm{~s}) \mathrm{P}_{1}(\mathrm{~s}) \\
& \overline{\mathrm{P}_{16}}(\mathrm{~s})=\mathrm{g}_{16}(\mathrm{~s}) \mathrm{P}_{1}(\mathrm{~s}) \\
& \overline{\mathrm{P}_{17}}(\mathrm{~s})=\mathrm{g}_{17}(\mathrm{~s}) \mathrm{P}_{1}(\mathrm{~s})
\end{aligned}
$$

where,

$$
\begin{gathered}
\mathrm{g}_{1}(\mathrm{~s})=\left(\mathrm{s}+\lambda_{\mathrm{a}}+\lambda_{\mathrm{b}}+\lambda_{1}+\lambda_{2}+\lambda\right)-\mu_{1}\left(\mathrm{~g}_{2}(\mathrm{~s})+\mathrm{g}_{3}(\mathrm{~s})\right) \\
-\mu\left(\mathrm{g}_{4}(\mathrm{~s})+\mathrm{g}_{8}(\mathrm{~s})+\mathrm{g}_{7}(\mathrm{~s})+\mathrm{g}_{8}(\mathrm{~s})+\mathrm{g}_{9}(\mathrm{~s})+\mathrm{g}_{10}(\mathrm{~s})+\mathrm{g}_{13}(\mathrm{~s})\right. \\
\left.\mathrm{g}_{14}(\mathrm{~s})+\mathrm{g}_{15}(\mathrm{~s})+\mathrm{g}_{16}(\mathrm{~s})+\mathrm{g}_{17}(\mathrm{~s})\right)-\mu_{2}\left(\mathrm{~g}_{6}(\mathrm{~s})+\mathrm{g}_{12}(\mathrm{~s})\right) \\
\mathrm{g}_{2}(\mathrm{~s})=\frac{\lambda_{\mathrm{a}}}{\mathrm{s}+\lambda+\lambda_{\mathrm{b}}+\mu_{1}}, \mathrm{~g}_{3}(\mathrm{~s})=\frac{\lambda_{\mathrm{b}}}{\mathrm{s}+\lambda+\lambda_{\mathrm{a}}+\mu_{1}} \\
\mathrm{~g}_{4}(\mathrm{~s})=\lambda_{\mathrm{a}} \lambda_{\mathrm{b}}\left(\frac{1}{\left(\mathrm{~s}+\lambda+\lambda_{\mathrm{a}}+\mu_{1}\right.}+\frac{1}{\mathrm{~s}+\lambda+\lambda_{\mathrm{b}}+\mu_{1}}\right) \frac{1}{\mathrm{~s}+\mu} \\
\mathrm{g}_{6}(\mathrm{~s})=\frac{\lambda_{1}}{\left(\mathrm{~s}+\lambda+\lambda_{\mathrm{b}}+\lambda_{\mathrm{a}}+\lambda_{2}+v\right)\left(\mathrm{s}+\lambda+\lambda_{2}+\lambda_{\mathrm{b}}+\mu_{2}\right)} \\
\mathrm{g}_{5}(\mathrm{~s})=\frac{\lambda_{1} \lambda_{\mathrm{a}}}{\mathrm{s}+\lambda+\lambda_{\mathrm{b}}+\lambda_{\mathrm{a}}+\lambda_{2}+v} \\
\mathrm{~g}_{7}(\mathrm{~s})=\frac{\lambda_{1} \lambda_{\mathrm{a}} \lambda_{\mathrm{b}}}{\left(\mathrm{s}+\lambda+\lambda_{\mathrm{b}}+\lambda_{\mathrm{a}}+\lambda_{2}+v\right)} \\
\mathrm{g}_{10}(\mathrm{~s})=\frac{\left(\mathrm{s}+\lambda+\lambda_{2}+\lambda_{\mathrm{b}}+\mu_{2}\right)(\mathrm{s}+\mu)}{\left(\mathrm{s}+\lambda+\lambda_{\mathrm{b}}+\lambda_{\mathrm{a}}+\lambda_{2}+v\right)(\mathrm{s}+\mu)} \\
\lambda_{1} \lambda_{\mathrm{a}} \lambda_{2} \\
\frac{\lambda_{1}(\mathrm{~s})=\frac{\lambda_{\mathrm{b}}}{\left(\mathrm{s}+\lambda+\lambda_{\mathrm{b}}+\lambda_{\mathrm{a}}+\lambda_{2}+v\right)}}{\left(\mathrm{s}+\lambda+\lambda_{2}+\lambda_{\mathrm{b}}+\mu_{2}\right)(\mathrm{s}+\mu)} \\
\lambda_{1} \lambda_{2}
\end{gathered}
$$

$$
\begin{gathered}
\mathrm{g}_{11}(\mathrm{~s})=\frac{\lambda_{2}}{\mathrm{~s}+\lambda+\lambda_{\mathrm{b}}+\lambda_{\mathrm{a}}+\lambda_{1}+v} \\
\mathrm{~g}_{12}(\mathrm{~s})=\frac{\lambda_{2} \lambda_{\mathrm{b}}}{\left(\mathrm{s}+\lambda+\lambda_{\mathrm{b}}+\lambda_{\mathrm{a}}+\lambda_{1}+\mathrm{v}\right)\left(\mathrm{s}+\lambda+\lambda_{1}+\lambda_{\mathrm{a}}+\mu_{2}\right)} \\
\mathrm{g}_{13}(\mathrm{~s})=\frac{\lambda_{1} \lambda_{2} \lambda_{\mathrm{b}}}{\left(\mathrm{s}+\lambda+\lambda_{\mathrm{b}}+\lambda_{\mathrm{a}}+\lambda_{1}+v\right)} \\
\mathrm{g}_{14}(\mathrm{~s})=\frac{\left(\mathrm{s}+\lambda+\lambda_{1}+\lambda_{\mathrm{a}}+\mu_{2}\right)(\mathrm{s}+\mu)}{\left(\mathrm{s}+\lambda+\lambda_{\mathrm{b}}+\lambda_{\mathrm{a}}+\lambda_{1}+v\right)} \\
\mathrm{g}_{15}(\mathrm{~s})=\frac{\left(\mathrm{s}+\lambda+\lambda_{1}+\lambda_{\mathrm{a}}+\mu_{2}\right)(\mathrm{s}+\mu)}{\left(\mathrm{s}+\lambda+\lambda_{\mathrm{b}}+\lambda_{\mathrm{a}}+\lambda_{1}+v\right)(\mathrm{s}+\mu)} \\
\mathrm{g}_{16}(\mathrm{~s})=\frac{\lambda_{\mathrm{a}} \lambda_{2}}{\left(\mathrm{~s}+\lambda+\lambda_{\mathrm{b}}+\lambda_{\mathrm{a}}+\lambda_{1}+v\right)(\mathrm{s}+\mu)} \\
\mathrm{g}_{17}(\mathrm{~s})=\frac{\lambda}{(\mathrm{s}+\mu)}\left\{\begin{array}{l}
\mathrm{g}_{2}(\mathrm{~s})+\mathrm{g}_{3}(\mathrm{~s})+\mathrm{g}_{5}(\mathrm{~s})+ \\
\mathrm{g}_{6}(\mathrm{~s})+\mathrm{g}_{11}(\mathrm{~s})+\mathrm{g}_{12}(\mathrm{~s})
\end{array}\right\}
\end{gathered}
$$

OPERATIONAL AVAILABILITY AND NON AVAILABILITY

The Laplace Transform of the probabilities that the system is in operable and down state at time $t$, are given as follows:

$$
\begin{aligned}
\overline{\mathrm{P}}_{\text {up }}(\mathrm{s})= & \overline{\mathrm{P}}_{1}(\mathrm{~s})+\overline{\mathrm{P}}_{2}(\mathrm{~s})+\overline{\mathrm{P}}_{3}(\mathrm{~s})+\overline{\mathrm{P}}_{5}(\mathrm{~s})+ \\
& \overline{\mathrm{P}}_{6}(\mathrm{~s})+\overline{\mathrm{P}}_{11}(\mathrm{~s})+\overline{\mathrm{P}}_{12}(\mathrm{~s}) \\
= & \overline{\mathrm{P}}_{1}(\mathrm{~s})\left\{\begin{array}{l}
1+\mathrm{g}_{2}(\mathrm{~s})+\mathrm{g}_{3}(\mathrm{~s})+\mathrm{g}_{5}(\mathrm{~s})+ \\
\mathrm{g}_{6}(\mathrm{~s})+\mathrm{g}_{11}(\mathrm{~s})+\mathrm{g}_{12}(\mathrm{~s})
\end{array}\right\} \\
& \overline{\mathrm{P}}_{\text {down }}(\mathrm{s})=1-\overline{\mathrm{P}}_{\text {up }}(\mathrm{s})
\end{aligned}
$$

Reliability: The reliability is given by:

$\mathrm{R}(\mathrm{t})=\mathrm{m}_{1} \mathrm{e}^{-\mathrm{q}_{1} \mathrm{t}}+\mathrm{m}_{2} \mathrm{e}^{-\mathrm{q}_{2} \mathrm{t}}+\mathrm{m}_{3} \mathrm{e}^{-\mathrm{q}_{3} \mathrm{t}}+\mathrm{m}_{5} \mathrm{e}^{-\mathrm{q}_{\mathrm{s}} \mathrm{t}}+\mathrm{m}_{7} \cdot \mathrm{e}^{-\mathrm{q}_{7} \mathrm{t}}$ where,

$$
\begin{aligned}
& \mathrm{q}_{1}=\lambda_{\mathrm{a}}+\lambda_{\mathrm{b}}+\lambda_{1}+\lambda_{2}+\lambda, \mathrm{q}_{2}=\lambda_{\mathrm{b}}+\lambda, \mathrm{q}_{3}=\lambda_{\mathrm{a}}+\lambda \\
& \mathrm{q}_{5}=\lambda_{\mathrm{b}}+\lambda_{2}+\lambda, \mathrm{q}_{7}=\lambda_{\mathrm{a}}+\lambda_{1}+\lambda \\
& \mathrm{m}_{1}=\mathrm{m}_{8}+\mathrm{m}_{9}-1-\mathrm{m}_{2}-\mathrm{m}_{3}
\end{aligned}
$$




$$
\begin{aligned}
& \mathrm{m}_{2}=\frac{\lambda_{\mathrm{a}}}{\lambda_{\mathrm{a}}+\lambda_{1}+\lambda_{2}}, \mathrm{~m}_{3}=\frac{\lambda_{\mathrm{b}}}{\lambda_{\mathrm{b}}+\lambda_{1}+\lambda_{2}}, \mathrm{~m}_{5}=\frac{\lambda_{1}}{\lambda_{\mathrm{a}}+\lambda_{1}}, \\
& \mathrm{~m}_{7}=\frac{\lambda_{2}}{\lambda_{\mathrm{b}}+\lambda_{2}} \mathrm{~m}_{8}=\frac{\lambda_{\mathrm{a}}}{\lambda_{\mathrm{a}}+\lambda_{1}}, \mathrm{~m}_{9}=\frac{\lambda_{\mathrm{b}}}{\lambda_{\mathrm{b}}+\lambda_{2}}
\end{aligned}
$$

MTTF: The mean time to system failure is given by:

$$
\text { MTTF }=\int_{0}^{\infty} \mathrm{R}(\mathrm{t}) \mathrm{dt}=\frac{\mathrm{m}_{1}}{\mathrm{q}_{1}}+\frac{\mathrm{m}_{2}}{\mathrm{q}_{2}}+\frac{\mathrm{m}_{3}}{\mathrm{q}_{3}}+\frac{\mathrm{m}_{5}}{\mathrm{q}_{5}}+\frac{\mathrm{m}_{7}}{\mathrm{q}_{7}}
$$

\section{NUMERICAL ILLUSTRATIONS}

\section{Analysis of availability: Setting}

$$
\begin{aligned}
\lambda_{\mathrm{a}} & =0.001, \lambda_{\mathrm{b}}=0.002, \lambda_{1}=0.001, \\
\lambda_{2} & =0.002, \lambda=0.009, \\
& \mathrm{v}=0.95, \mu_{1}=0.92, \mu_{2}=0.86
\end{aligned}
$$

in the Eq. 70 and then taking the inverse Laplace transform, the operational availability is obtained as:

$$
\begin{aligned}
P_{\text {up }}(t)= & z_{1} e^{-n_{1} t}+z_{2} e^{-n_{2} t}+z_{3} e^{-n_{3} t}+ \\
& z_{4} e^{-n_{4} t}+z_{5} e^{-n_{5} t}+z_{6} e^{-n_{6} t}+z_{7} e^{-n_{7} t}
\end{aligned}
$$

where

$$
\begin{aligned}
& \mathrm{n}_{1}=\lambda_{\mathrm{a}}+\lambda_{\mathrm{b}}+\lambda_{1}+\lambda_{2}+\lambda, \mathrm{n}_{2}=\lambda_{\mathrm{b}}+\lambda+\mu_{1}(\mathrm{x}), \\
& \mathrm{n}_{3}=\lambda_{\mathrm{a}}+\lambda+\mu_{1}(\mathrm{x}) \\
& \mathrm{n}_{4}=\lambda_{\mathrm{a}}+\lambda_{\mathrm{b}}+\lambda_{2}+\lambda+v, \mathrm{n}_{5}=\lambda_{\mathrm{b}}+\lambda+\lambda_{2}+\mu_{2}(\mathrm{y})
\end{aligned}
$$$$
\mathrm{n}_{6}=\lambda_{\mathrm{a}}+\lambda_{\mathrm{b}}+\lambda_{1}+\lambda+v, \mathrm{n}_{7}=\lambda_{\mathrm{a}}+\lambda+\lambda_{1}+\mu_{2}(\mathrm{y})
$$

$$
\begin{aligned}
& \mathrm{z}_{1}=1-\frac{\lambda_{\mathrm{a}}}{\mathrm{n}_{1}-\mathrm{n}_{2}}-\frac{\lambda_{\mathrm{b}}}{\mathrm{n}_{1}-\mathrm{n}_{3}}-\frac{\lambda_{1}}{\mathrm{n}_{1}-\mathrm{n}_{4}}+ \\
& \frac{\lambda_{\mathrm{a}} \lambda_{1}}{\left(\mathrm{n}_{4}-\mathrm{n}_{1}\right)\left(\mathrm{n}_{5}-\mathrm{n}_{1}\right)}-\frac{\lambda_{2}}{\mathrm{n}_{1}-\mathrm{n}_{6}}+\frac{\lambda_{\mathrm{b}} \lambda_{2}}{\left(\mathrm{n}_{6}-\mathrm{n}_{1}\right)\left(\mathrm{n}_{7}-\mathrm{n}_{1}\right)}
\end{aligned}
$$$$
\mathrm{z}_{2}=\frac{\lambda_{\mathrm{a}}}{\mathrm{n}_{1}-\mathrm{n}_{2}}, \mathrm{z}_{3}=\frac{\lambda_{\mathrm{b}}}{\mathrm{n}_{1}-\mathrm{n}_{3}},
$$$$
\mathrm{z}_{3}=\frac{\lambda_{1}}{\mathrm{n}_{1}-\mathrm{n}_{4}}+\frac{\lambda_{\mathrm{a}} \lambda_{1}}{\left(\mathrm{n}_{1}-\mathrm{n}_{4}\right)\left(\mathrm{n}_{5}-\mathrm{n}_{4}\right)}
$$

$$
\begin{gathered}
\mathrm{z}_{5}=\frac{\lambda_{\mathrm{a}} \lambda_{1}}{\left(\mathrm{n}_{4}-\mathrm{n}_{5}\right)\left(\mathrm{n}_{1}-\mathrm{n}_{5}\right)}, \mathrm{z}_{6}=\frac{\lambda_{2}}{\mathrm{n}_{1}-\mathrm{n}_{6}}+\frac{\lambda_{\mathrm{b}} \lambda_{2}}{\left(\mathrm{n}_{7}-\mathrm{n}_{6}\right)\left(\mathrm{n}_{1}-\mathrm{n}_{6}\right)} \\
\mathrm{z}_{7}=\frac{\lambda_{\mathrm{b}} \lambda_{2}}{\left(\mathrm{n}_{6}-\mathrm{n}_{7}\right)\left(\mathrm{n}_{1}-\mathrm{n}_{7}\right)}
\end{gathered}
$$

Substituting different values of $t$ is equation (74) one may obtain Table 1 and Fig. 1.

Reliability analysis: Setting $\lambda_{\mathrm{a}}=0.001, \lambda_{\mathrm{b}}=$ $0.002, \lambda_{1}=0.011, \lambda_{2}=0.015, \lambda=0.05$ in the Eq. 72 one may obtain the variations in reliability of the system with time as shown in Table 2 and Fig. 2.

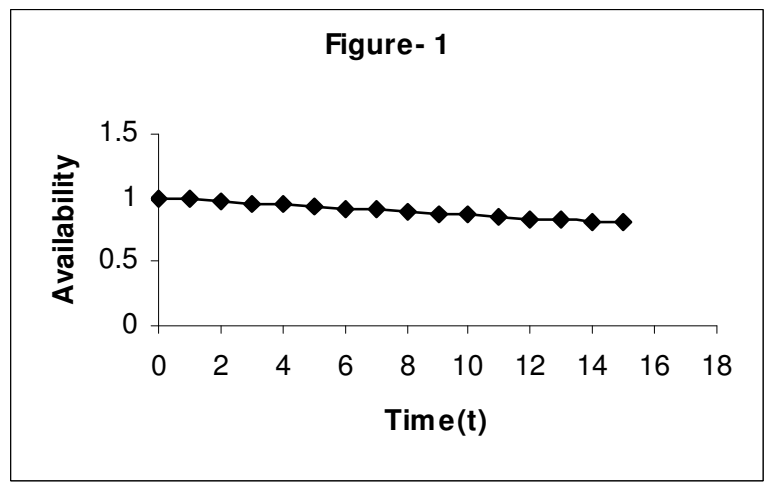

Fig. 1: Availability v/s Time

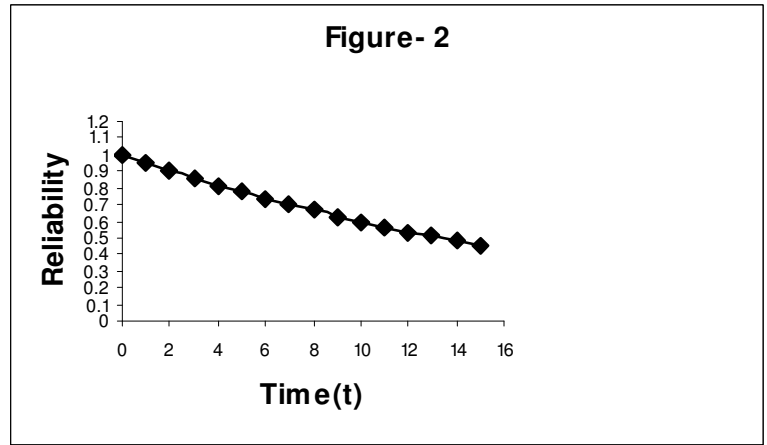

Fig. 2: Reliability v/s Time

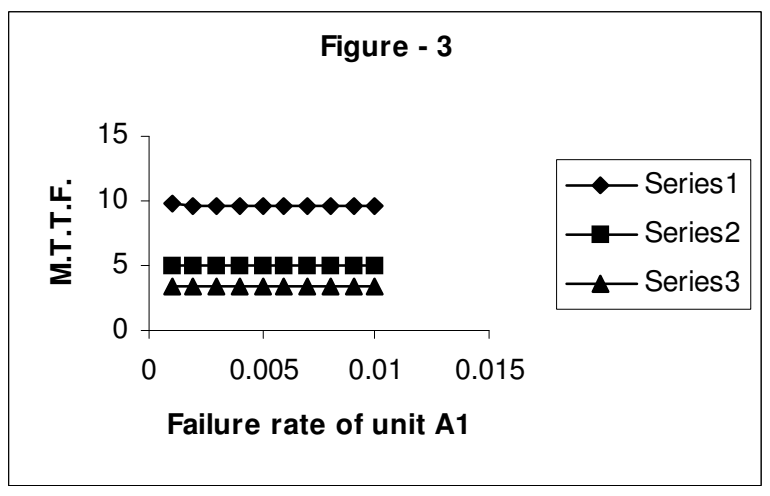

Fig. 3: M.T.T.F. v/s Failure rate of unit A1 
J. Math. \& Stat., 3 (4): 211-219, 2007

\begin{tabular}{lc} 
Table 1: Variation of availability with time \\
\hline Time (t) & Availability [Pup (t)] \\
\hline 0 & 0.999982 \\
1 & 0.988951 \\
2 & 0.975725 \\
3 & 0.96178 \\
4 & 0.947687 \\
5 & 0.933666 \\
6 & 0.9198 \\
7 & 0.906119 \\
8 & 0.892634 \\
9 & 0.879346 \\
10 & 0.866255 \\
11 & 0.853359 \\
12 & 0.840654 \\
13 & 0.828138 \\
14 & 0.815809 \\
15 & 0.803663 \\
\hline
\end{tabular}

Table 2; Variation of reliability with time

\begin{tabular}{lc}
\hline Time $(\mathrm{t})$ & Reliability[R $(\mathrm{t})]$ \\
\hline 0 & 1 \\
1 & 0.951056 \\
2 & 0.904185 \\
3 & 0.859332 \\
4 & 0.816437 \\
5 & 0.77544 \\
6 & 0.736279 \\
7 & 0.698895 \\
8 & 0.663224 \\
9 & 0.629205 \\
10 & 0.596777 \\
11 & 0.565881 \\
12 & 0.536456 \\
13 & 0.508444 \\
14 & 0.481789 \\
\hline
\end{tabular}

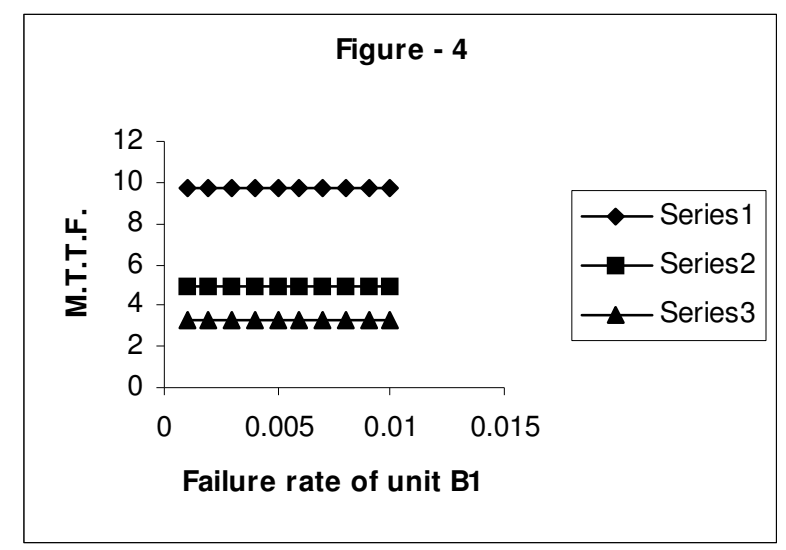

Fig. 4: M.T.T.F. v/s Failure rate of unit B1

Table 3: Variation of M. T. T. F. with failure rate of $A_{1}$ unit

\begin{tabular}{|c|c|c|c|}
\hline \multicolumn{4}{|c|}{ M.T.T.F. } \\
\hline 0.001 & 9.712430 & 4.955933 & 3.319280 \\
\hline 0.002 & 9.699658 & 4.953789 & 3.318573 \\
\hline 0.003 & 9.687316 & 4.951683 & 3.317875 \\
\hline
\end{tabular}

\begin{tabular}{llll}
0.004 & 9.675385 & 4.949614 & 3.317185 \\
0.005 & 9.66385 & 4.947581 & 3.316503 \\
0.006 & 9.652699 & 4.94585 & 3.31583 \\
0.007 & 9.641916 & 4.943624 & 3.315165 \\
0.008 & 9.63149 & 4.941698 & 3.314507 \\
0.009 & 9.621406 & 4.939805 & 3.313858 \\
0.001 & 9.712430 & 4.955933 & 3.319280 \\
\hline
\end{tabular}

\section{MTTF analysis:}

- $\quad$ Setting the values $\lambda_{2}=0.02, \lambda_{1}=0.01, \lambda_{b}=0.002$ and taking different values of $\lambda_{\mathrm{a}}$ in the Eq. 73 one may obtain the variations of MTTF of the system against the failure rate of unit $A_{1},\left(\lambda_{a}\right)$ as shown in Table 3 and Fig. 3.

- Setting the values $\lambda_{2}=0.02, \lambda_{1}=0.01, \lambda_{\mathrm{a}}=0.001$ and taking different values of $\lambda_{b}$ in the Eq. 73 one may obtain the variations of M.T.T.F. of the system against the failure rate of unit $\mathrm{B}_{1}\left(\lambda_{\mathrm{b}}\right)$ as shown in Table 4 and Fig. 4.

- $\quad$ Setting the values $\lambda_{2}=0.02, \lambda_{a}=0.001, \lambda_{b}=0.002$ and taking different values of $\lambda$ in the Eq. 73 one may

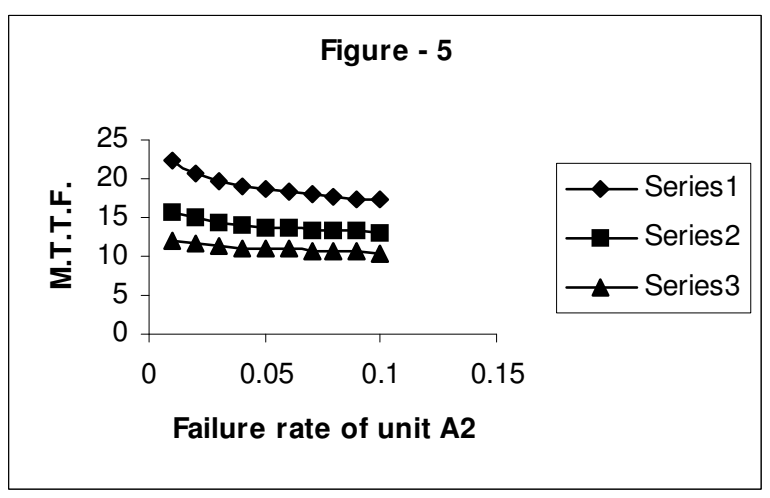

Fig. 5: M.T.T.F. v/s Failure rate of unit A2

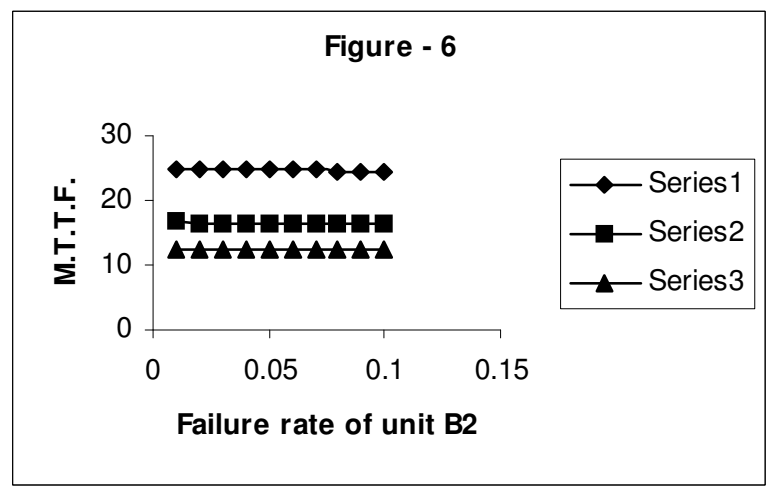

Fig. 6: M.T.T.F. v/s Failure rate of unit B2 
J. Math. \& Stat., 3 (4): 211-219, 2007

Table 4: Variation of M. T. T. F. with failure rate of $B_{1}$ unit

\begin{tabular}{|c|c|c|c|}
\hline \multicolumn{2}{|c|}{ M.T.T.F. } & & \\
\hline 0.001 & 9.716869 & 4.956836 & 3.319596 \\
\hline 0.002 & 9.71243 & 4.955933 & 3.31928 \\
\hline 0.003 & 9.708154 & 4.955048 & 3.318968 \\
\hline 0.004 & 9.704035 & 4.954179 & 3.318661 \\
\hline 0.005 & 9.700066 & 4.953325 & 3.318357 \\
\hline 0.006 & 9.696241 & 4.952488 & 3.318056 \\
\hline 0.007 & 9.692557 & 4.951665 & 3.31776 \\
\hline 0.008 & 9.689009 & 4.950858 & 3.317467 \\
\hline 0.009 & 9.68559 & 4.950065 & 3.317177 \\
\hline 0.01 & 9.682298 & 4.949286 & 3.316892 \\
\hline
\end{tabular}

Table 5: Variation of M. T. T. F. with failure rate of $A_{2}$ unit

\begin{tabular}{|c|c|c|c|}
\hline & M.T.T.F. & & \\
\hline 0.01 & 22.274481 & 15.618896 & 11.988547 \\
\hline 0.02 & 20.759512 & 14.948854 & 11.634022 \\
\hline 0.03 & 19.765066 & 14.465042 & 11.362288 \\
\hline 0.04 & 19.074417 & 14.103583 & 11.149179 \\
\hline 0.05 & 18.573645 & 13.825957 & 10.978786 \\
\hline 0.06 & 18.197981 & 13.60778 & 10.840281 \\
\hline 0.07 & 17.908289 & 13.432982 & 10.726077 \\
\hline 0.08 & 17.679733 & 13.290612 & 10.630732 \\
\hline 0.09 & 17.495913 & 13.17299 & 10.550251 \\
\hline 0.1 & 17.345627 & 13.0746 & 10.481655 \\
\hline
\end{tabular}

obtain the variations of MTTF of the system against the failure rate of unit $A_{1}\left(\lambda_{a}\right)$ as shown in Table 5 and Fig. 5.

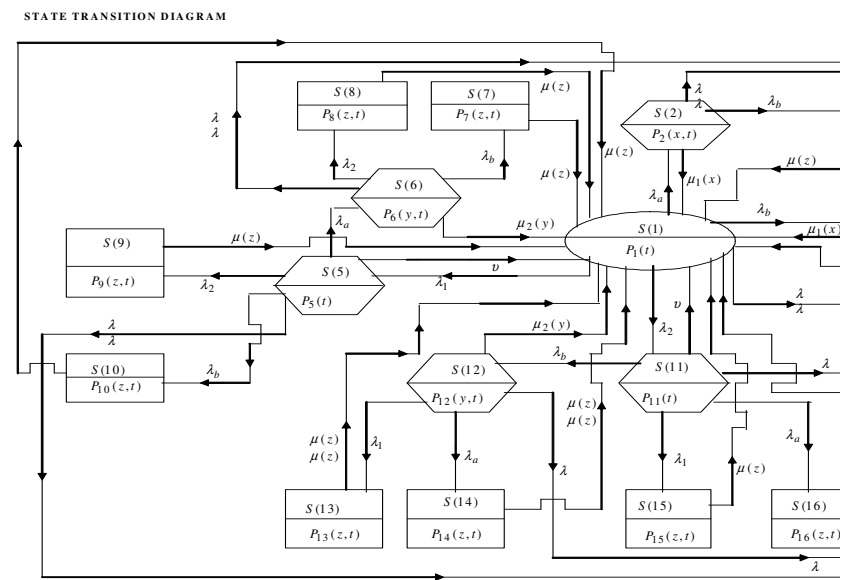

Fig. 7: STATE TRANSITION DIAGRAM

Table 6: Variation of M. T. T. F. with failure rate of $B_{2}$ unit

\begin{tabular}{|c|c|c|c|}
\hline \multicolumn{2}{|c|}{ M.T.T.F. } & \multirow[b]{2}{*}{16.614389} & \multirow[b]{2}{*}{12.47681} \\
\hline 0.01 & 24.839481 & & \\
\hline 0.02 & 24.768173 & 16.587769 & 12.464078 \\
\hline 0.03 & 24.716404 & 16.566874 & 12.453601 \\
\hline 0.04 & 24.677107 & 16.550035 & 12.444826 \\
\hline 0.05 & 24.646261 & 16.536179 & 12.437371 \\
\hline 0.06 & 24.621405 & 16.524572 & 12.430959 \\
\hline
\end{tabular}

\begin{tabular}{llll}
0.07 & 24.600948 & 16.514713 & 12.425385 \\
0.08 & 24.58382 & 16.506235 & 12.420496 \\
0.09 & 24.569265 & 16.498861 & 12.416171 \\
0.1 & 24.556746 & 16.492395 & 12.412319 \\
\hline
\end{tabular}

- $\quad$ Setting the values $\lambda_{1}=0.01, \lambda_{a}=0.001, \lambda_{b}=0.002$ and taking different values of $\lambda$ in the Eq. 73 one may obtain the variations of MTTF of the system against the failure rate of unit $B_{2}\left(\lambda_{2}\right)$ as shown in Table 6 and Fig. 6.

\section{CONCLUSION}

The concept of redundancy of the generating unit can be applied to the areas where the electricity requirements are increasing at an alarming rate. The findings in Table 1 and 2 depicts that the system is available and reliable for a longer time period. The failures are considered to occur purely by chance for a component which is operating within its useful life period. So, under these conditions the calculations shown in Table 3-6 shows apparently that the failure rate is the reciprocal of the mean time to system failure (MTTF).

\section{ACKNOWLEDGMENT}

Authors are grateful to Rear Admiral Dr. Ajay Sharma (Director General) and Prof. G.S. Sandhu (Director), of Krishna Institute of Engineering \& Technology, Ghaziabad (India) for their constant encouragement, and providing necessary facilities (including financial support) to carry out the present work.

\section{NOTATIONS}

Failure and constant repair rate of unit $\mathrm{A}_{2}$ or $\mathrm{B}_{2}$ $(i=1,2)$.

$\lambda_{\mathrm{j}}, \mu_{1}(\mathrm{x})$ : Failure and repair rate of unit $\mathrm{A}_{2}$ or $\mathrm{B}_{1}(\mathrm{j}=\mathrm{a}, \mathrm{b})$.

$\lambda: \quad$ Failure rate of subsystem $\mathrm{C}$.

$\mu(\mathrm{z})$ : $\quad$ Repair rate when the system is in failed state.

$\mu_{2}(y)$ : Repair rate of both units of subsystem $A$ or subsystem B.

$\mathrm{P}_{1}(\mathrm{t})$ : Probability that the system is in operable state at time t.

$\mathrm{P}_{\mathrm{i}}(\mathrm{x}, \mathrm{t}) \Delta$ : Probability that the system is in degraded state at time $\mathrm{t}$ and elapsed repair time lies between $\mathrm{x}$ and $\mathrm{x}+\Delta$, where $9 \mathrm{i}=2,3$ )

$\mathrm{P}_{\mathrm{j}}(\mathrm{y}, \mathrm{t}) \Delta$ : Probability that the system is in degraded state at time $t$ and elapsed repair time lies between $y$ and $\mathrm{y}+\Delta$, where $(\mathrm{j}=6,12)$ 
$\mathrm{P}_{\mathrm{k}}(\mathrm{z}, \mathrm{t}) \Delta$ : Probability that the system is in failed state at time $\mathrm{t}$ and elapsed repair time lies between $\mathrm{z}$ and $\mathrm{z}+\Delta$, where $(\mathrm{k}=4,7,8,9,10,113,14,15$, 16, 17)

$P_{m}(t): \quad$ Probability that the system is in degraded state at time $\mathrm{t}$ where $(\mathrm{m}=5,11)$ $\int=\int_{0}^{\infty}$, Otherwise stated.

$\bigcirc_{\text {:Operable }} \square_{\text {:Degraded }} \square$ : Failed

\section{REFERENCES}

1 Brombacher, A.C., 2005. Reliability in strongly innovative products: A threat or a challenge? Reliability Eng. Syst. Safety, 88: 125.

2 Cassanelli, G., G. Mura, F. Cesaretti, M. Vanzi and F. Fantini, 2005. Reliability predictions in electronic. Ind. Applic., 45: 1321-1326.

3 Reliability Engineering. Balagurusamy, E. (Ed.), Tata McGraw-Hill Publishing Company Ltd.

4 Generation of Electrical Energy. by Gupta, B.R. (Ed.), Eurasis Publishing House (Pvt.) Ltd. 